“ (C) 2017 IEEE. Personal use of this material is permitted. Permission from IEEE must be obtained for all other uses, in any current or future media, including

reprinting/republishing this material for advertising or promotional purposes, creating new collective works, for resale or redistribution to servers or lists, or reuse of any copyrighted component of this work in other works." 


\title{
Scalable quantum tomography in a photonic chip
}

\author{
James Titchener ${ }^{1,2, *}$, Markus Gräfe ${ }^{2}$, René Heilmann², Alexander S. Solntsev ${ }^{1}$, Alexander Szameit ${ }^{2}$, and \\ Andrey A. Sukhorukov ${ }^{1}$ \\ ${ }^{1}$ Nonlinear Physics Centre, Research School of Physics and Engineering, Australian National University, Canberra, ACT 2601, Australia \\ ${ }^{2}$ Institute of Applied Physics, Abbe Center of Photonics, Friedrich-Schiller-Universität Jena, Max-Wien-Platz 1, Jena 07743, Germany \\ *james.titchener@anu.edu.au
}

\begin{abstract}
We formulate a method of quantum tomography that scales linearly with the number of photons and involves only one optical transformation. We demonstrate it experimentally for twophoton entangled states using a special photonic chip.

OCIS codes: (130.3120) Integrated optics devices; (270.5585) Quantum information and processing.
\end{abstract}

Full characterization of quantum states is increasingly important as ever more complex quantum systems are realized. Quantum state tomography of these systems is a difficult task because individual measurements only give partial information about the underlying density matrix of the system. Thus, conventionally the measurement apparatus must be reconfigured to take multiple measurements in order to fully determine the density matrix. It is accepted for an $N$-qubit system that an exponentially increasing number of measurements are needed, requiring $4^{N}$ different configurations of the optical setup [1]. As a consequence, the complexity of typical quantum state tomography grows exponentially with increasing photon number, which makes characterizing large quantum systems highly challenging.

We formulate a concept of on-chip quantum tomography where the complexity scales linearly with the number of photons. This is achieved by doing away with the need to reconfigure the measurement setup, and instead using a single specially designed non-reconfigurable linear optical circuit. Crucially, the number of waveguides required in this circuit scales linearly with the number of photons in the state to be measured. Furthermore, our approach is simple and robust, and we demonstrate it experimentally with a specially designed photonic chip.

We explain our method for the case of $N$ photons in an arbitrary pure or mixed state featuring spatial quantum entanglement between two input ports, although a larger number of ports can be considered as well [2]. The quantum photon state is described by a density matrix, $\hat{\rho}_{i n}$, which has $4^{N}$ elements. We then apply a single (fixed) linear transformation $\widehat{U}$ to map the quantum state from two inputs to a larger number of output waveguides, $M$, as illustrated in Fig. 1(a). Then, we obtain information about the quantum state by measuring coincidences $\Gamma$ in the arrival time of photons to different combinations of the output single-photon detectors. Then, the reconstruction of quantum state is possible if the number of correlations is larger than the number of unknown density matrix elements. This condition is graphed in Fig. 1(b), showing that the number of output ports scales linearly with the number of photons.
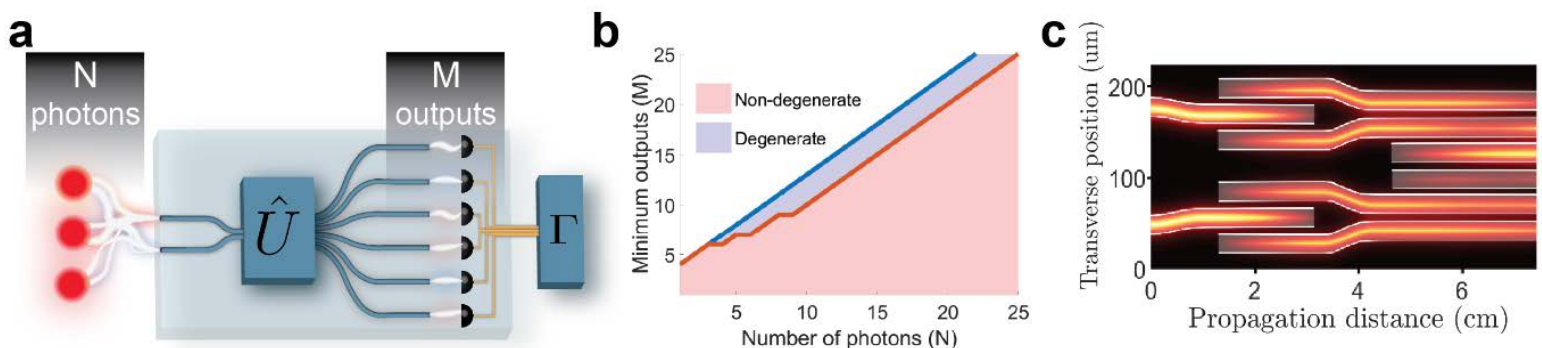

Fig. 1. (a) Conceptual diagram of on-chip quantum tomography based on a single optical transformation (b) Scaling of the number of waveguides and single photon detectors required for tomography of increasingly many photons. (c) Experimentally realized waveguide based tomography chip showing the probability amplitude of a single photon $(815 \mathrm{~nm})$ coupled into the chip (input superposition state $|0\rangle+i|1\rangle$ ).

We experimentally demonstrate our approach by performing tomography of spatially entangled two-photon mixed states using a specially designed on-chip laser-written waveguide circuit [3]. Generally, characterization of such a state would be achieved using a reconfigurable measurement setup [4], however we demonstrate that full tomography is possible with just the static waveguide circuit in Fig. 1(c). The circuit takes any two-photon mixed quantum state and transforms it to a sparse two-photon state at the six output waveguides. The circuit was specially optimized to allow full reconstruction of the input density matrix just by measuring the output two-photon coincidences with standard single-photon on-off detectors. The circuit was also optimized to make the tomographic reconstruction highly robust to measurement errors, this was done by minimizing its condition number of the optical 
transformation. The experimentally realized device has a condition number of 5 , in contrast to $\sim 10$ for typical approaches to tomography [1], meaning our chip is more robust to experimental errors.

To test the performance of the device we prepared a range of different two-photon entangled quantum states and coupled them into the chip in Fig. 1(c). Measuring the 2-photon correlations at the output of the chip then allowed reconstruction of the input photon density matrix. For example, the measured correlations resulting from coupling a 2-photon N00N state into the chip are shown in Fig. 2(a). Using a simple and computationally scalable algorithm [5] we iteratively minimized the 'distance measure' between the measured correlations and the correlations produced by the input density matrix at each step. The progress of this minimization is shown in Fig. 2(b) for 1000 different realizations of the algorithm. The inset of Fig. 2(b) shows the resulting reconstruction of the input density matrix, which indeed is a N00N state with fidelity exceeding 94\%. Importantly, this is the first experimental realization of self-guided quantum tomography algorithm [5] for mixed two-photon states.

Our approach to quantum tomography based on a static circuit is well suited for reconstruction with high precision of the density matrix of a quantum state that is varying over time. We demonstrated this by observing the quantum-enhanced temporal variation of the phase in the density matrix of the N00N state to temperature and strain fluctuations in one-meter-long sections of optical fiber before coupling into the chip.

The same photon chip is also capable of tomography of 3-photon quantum states. To test this we used the experimentally determined transfer function of the chip to simulate the measurement of a 3-photon state. The simulated 3-photon correlations at the output of the chip for an input GHZ state are shown in Fig. 2(c). After adding Gaussian noise to the correlations, reconstruction of the input density matrix is performed. The real part of the reconstructed density matrix is shown in Fig. 2(d), which matches the expected GHZ state.
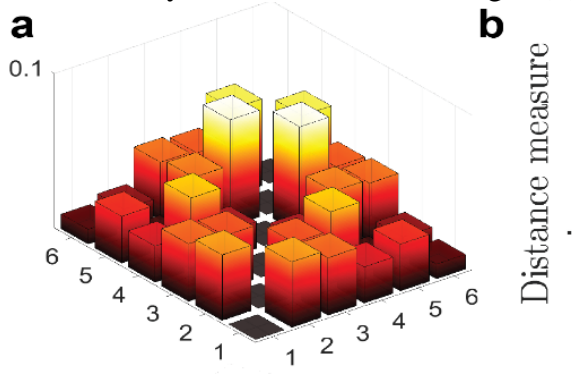

C

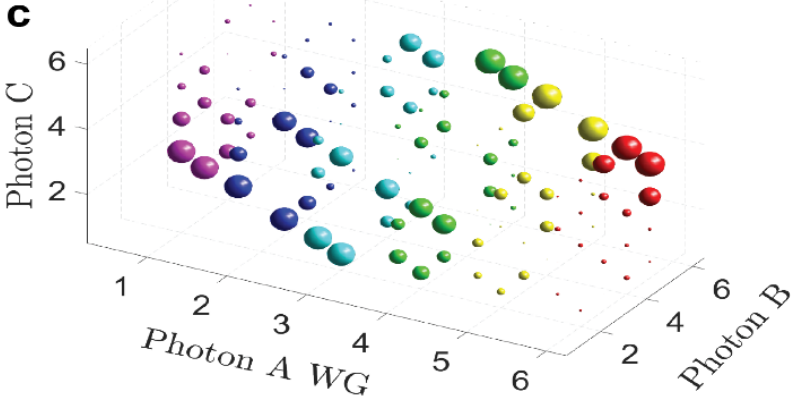

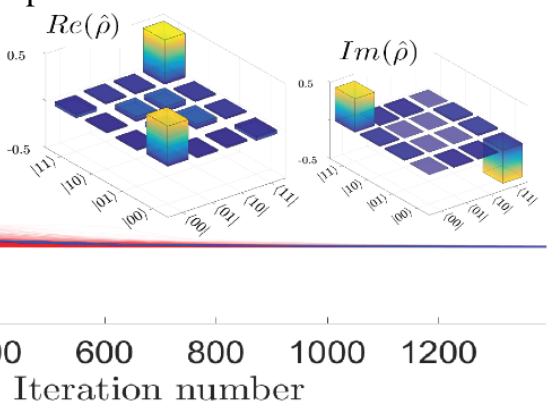

d

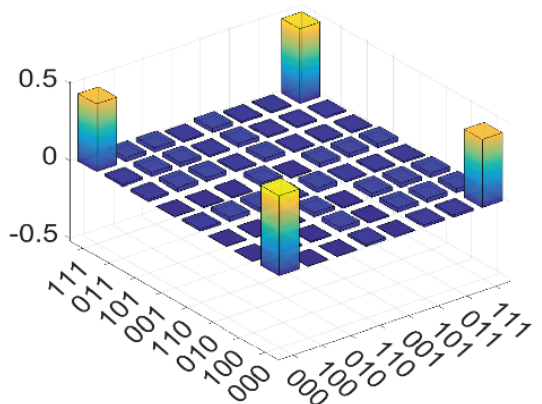

Fig. 2. (a) Experimentally measured two-photon coincidence probabilities after the device. (b) Minimization of discrepancy between guessed density matrix and observed correlations (1000 realizations in red). Inset shows the real and imaginary parts of the optimal density matrix produced at the convergence of iterations. (c) Simulated correlations for an input 3-photon GHZ state calculated using experimentally determined device transfer function. Diameter of each sphere gives the probability of detecting the 3-photons in different combinations of output waveguides. (d) Real part of 3-photon N00N state's density matrix reconstructed from correlations.

In conclusion, we have formulated and demonstrated experimentally a new concept for on-chip quantum state tomography, which does not require any reconfigurable elements, and is fundamentally more robust than standard tomography. This enables, in particular, stable imaging of density matrices that are changing in time. Furthermore, with our approach, the optical chip size scales linearly with the number of photons, whereas conventional tomography requires the measurement setup to be adjusted an exponentially increasing number of times.

\section{References}

[1] D. F. V. James, P. G. Kwiat, W. J. Munro, and A. G. White, “Measurement of qubits”, Phys. Rev. A 64, 052312 (2001).

[2] J. G. Titchener, A. S. Solntsev, and A. A. Sukhorukov, "Two-photon tomography using on-chip quantum walks," Opt. Lett. 41, 4079 (2016).

[3] T. Meany et al., "Laser written circuits for quantum photonics” Laser Phot. Rev. 9, 363 (2015)

[4] P. J. Shadbolt et al., "Generating, manipulating and measuring entanglement and mixture with a reconfigurable photonic circuit”, Nature Photonics 6, 45 (2012).

[5] C. Ferrie, “Self-Guided Quantum Tomography”, Phys. Rev. Lett. 113, 190404 (2014). 